$\begin{array}{ll}\text { Balkanologie } & \begin{array}{l}\text { Balkanologie } \\ \text { Revue d'études pluridisciplinaires }\end{array}\end{array}$

Vol. XIII, $n^{\circ}$ 1-2 | 2011

Volume XIII Numéro 1-2

\title{
Les « pyramides » de Bosnie-Herzégovine : une affaire de pseudo-archéologie dans le contexte bosnien
}

Irna

\author{
(2) OpenEdition \\ Journals \\ Édition électronique \\ URL : http://journals.openedition.org/balkanologie/2298 \\ DOI : 10.4000/balkanologie.2298 \\ ISSN : 1965-0582 \\ Éditeur \\ Association française d'études sur les Balkans (Afebalk)
}

Référence électronique

Irna, «Les « pyramides » de Bosnie-Herzégovine : une affaire de pseudo-archéologie dans le contexte bosnien », Balkanologie [En ligne], Vol. XIII, n 1-2 | 2011, mis en ligne le 15 décembre 2011, consulté le

17 décembre 2020. URL : http://journals.openedition.org/balkanologie/2298 ; DOI : https://doi.org/

10.4000/balkanologie.2298

Ce document a été généré automatiquement le 17 décembre 2020.

(c) Tous droits réservés 


\section{Les « pyramides » de Bosnie- Herzégovine : une affaire de pseudo- archéologie dans le contexte bosnien}

Irna

\section{NOTE DE L'ÉDITEUR}

L'auteure a tenu à garder l'anonymat. Pour toute mise en contact, veuillez vous adresser à la rédaction de Balkanologie ou prendre contact directement avec elle sur son site http://irna.lautre.net.

1 L'affaire des «pyramides » de Bosnie commence en avril 2006, lorsqu'une dépêche de l'Associated Press annonce au monde la découverte par l'Américano-Bosnien Semir Osmanagić, à Visoko en Bosnie Centrale, de gigantesques pyramides, plus hautes et plus anciennes que les pyramides d'Égypte. Bien que cette découverte ait suscité pendant quelque temps une activité fébrile dans la petite ville de Visoko, il est très vite évident aux yeux des archéologues et géologues, tant locaux qu'européens, que les " pyramides » ne sont que des collines aux versants vaguement triangulaires dont la forme doit tout à la nature, et les scientifiques se désintéressent très vite, pour la plupart, de la question.

2 Cependant, cinq ans plus tard, en 2011, les « pyramides » de Bosnie ne sont pas tombées dans l'oubli, du moins pour le grand public. Les fouilles entamées en 2006 sont toujours actives, renforcées chaque été par des fouilleurs bénévoles venus d'un peu partout, et financées, au moins partiellement, par les budgets publics de la ville, du canton, voire de la Fédération.

3 Le cas est assez unique pour qu'on s'y attarde quelque peu: si les affaires pseudoarchéologiques ne manquent pas dans les annales (Rennes-le-Château ${ }^{1}$, Glozel ${ }^{2}$, 
Stonehenge ${ }^{3}$, Cahokia Mounds ${ }^{4}$, Yonaguni ${ }^{5}$, multiples localisations de l'Atlantide - la liste est quasiment infinie !), c'est à ma connaissance la première fois dans l'histoire de la pseudo-archéologie qu'un tel projet, malgré son absence totale de validation scientifique, obtient à la fois une certaine forme de reconnaissance médiatique internationale et surtout autorisations de fouilles et financements publics ${ }^{6}$.

Cet article n'a pas prétention à être un article de recherche. L'auteur, géomorphologue et agrégée de Géographie, s'intéresse en amateur à l'histoire et l'archéologie des Balkans. C'est également en amateur qu'elle s'est penchée sur l'affaire des "pyramides» de Bosnie, et y consacre depuis 2006 un site critique (http:// irna.lautre.net), où divers aspects évoqués dans l'article sont exposés plus en détails. Sa connaissance de l'affaire repose pour l'essentiel sur le matériau publié sur internet depuis 2006, ainsi que sur quelques contacts personnels avec certains des protagonistes. Ce texte n'a donc ni prétention à l'exhaustivité, ni prétention à une quelconque expertise. Son but est d'essayer de présenter cette affaire peu commune et complexe, et de montrer qu'elle résulte de l'imbrication d'éléments très divers, parmi lesquels l'archéologie ne tient finalement qu'une place secondaire. Après avoir retracé l'historique de l'affaire, on montrera que le succès relatif de l'idée de pyramides préhistoriques en Bosnie tient à la fois à la présence de caractères spécifiques (géologiques et archéologiques) de la région de Visoko, à la stratégie adoptée par le promoteur des "pyramides", Semir Osmanagić, et enfin au contexte spécifique de la Bosnie des années 2000, hérité du conflit de 1992-1995.

\title{
1. La première pyramide européenne?
}

\begin{abstract}
«Depuis mercredi, des chercheurs mettent au jour des dalles de pierre géométriques, dont ils pensent qu'elles pourraient faire partie de la face d'une ancienne pyramide enterrée sous une colline gigantesque. Les archéologues et autres experts ont commencé à fouiller dans cette ville de Bosnie centrale la semaine dernière, pour confirmer la théorie du dirigeant de l'équipe : la colline de 750 mètres de haut cacherait une pyramide à degrés, la première trouvée en Europe. $»^{7}$
\end{abstract}

5 C'est par ces mots, repris quasiment à l'identique par de nombreux médias, que se répand hors de Bosnie la nouvelle de la découverte des «pyramides » de Visoko par Semir Osmanagić, un Américano-Bosnien vivant à Houston. Celui-ci a découvert le site de Visoko l'été précédent; lors d'une visite aux ruines de la forteresse médiévale qui surplombe Visoko, en compagnie du directeur du musée local, Senad Hodović, il est frappé d'une véritable révélation : la colline sur laquelle il se tient, Visočica, ainsi que celle qui lui fait face de l'autre côté de la rivière Fojnička, Plješevica, seraient en fait toutes deux des pyramides cachées par le sol et la végétation, qu'il compare aux pyramides du Soleil et de la Lune de Teotihuacan ${ }^{8}$. Dans les mois qui suivent, sur ses propres deniers (il est propriétaire d'une entreprise métallurgique assez prospère au Texas), il finance la réalisation de sondages géologiques sur Visočica - rebaptisée «Pyramide du Soleil». Il contacte un certain nombre de scientifiques, bosniens ou expatriés : Nadija Nukić, une géologue de Sarajevo, Amer Smailbegović, géophysicien américain qui accepte de lui fournir, par l'intermédiaire de son entreprise de télédétection, les images satellites de la région de Visoko. Considérant les résultats des premiers sondages ${ }^{9}$, qui révèlent une alternance de couches plus ou moins régulières, comme satisfaisants, il crée une fondation sans but lucratif (Fondation Parc 
archéologique: Pyramide du Soleil de Bosnie), et obtient pour cette fondation, par l'intermédiaire de Senad Hodović et du Musée de Visoko, les autorisations de fouilles nécessaires. Parallèlement, il entreprend la rédaction d'un livre, intitulé Bosanska Piramida Sunca (La Pyramide du Soleil de Bosnie), qui sera publié avant la fin de l'année $2005^{10}$.

\section{A. Ouverture du chantier archéologique}

6 L'ouverture du chantier de fouilles en avril 2006 est précédée d'une intense campagne de presse dans les médias de Bosnie, où il expose son hypothèse de pyramides préhistoriques ${ }^{11}$, et annonce la venue d'une équipe internationale de scientifiques (Suède, Grande-Bretagne, Australie, États-Unis) ${ }^{12}$.

7 Les fouilles commencent le 14 avril 2006 sur Visočica dans une ambiance, au dire des témoins, plus proche de celle d'une kermesse géante que de celle d'un chantier archéologique classique : centaines de volontaires prêts à manier la pelle, défilé des candidates au titre de Miss Bosnie, pot à café géant et concours de gâteaux - le tout sous l'objectif de la moitié des télévisions et photographes de presse du pays ${ }^{13}$. Les premiers résultats font l'objet de titres énormes dans la presse locale: «Dix sondages qui vont changer l'histoire » (San, 14 avril 2006), « Une nouvelle histoire s'écrit au cœur de la Bosnie » (San, 15 avril), «La face Nord de la Pyramide du Soleil est un triangle équilatéral » (Dnevni Avaz, 17 avril), «Au quatrième jour des fouilles, découverte de preuves matérielles de la construction des pyramides»(Dnevni Avaz, 18 avril), "Osmanagić : l'existence des pyramides est démontrée » (Dnevni List, 20 avril), «En Bosnie, la " mère de toutes les pyramides » ? (San, 20 avril).

8 Commence le défilé des hommes politiques venus admirer les dalles de la "pyramide» et surtout se faire photographier en compagnie de Semir Osmanagić par les nombreuses équipes de presse et de télévision, tandis que l'hôtel Hollywood de Visoko est rebaptisé hôtel Piramida Sunca, et que les habitants se reconvertissent en vendeurs de pizzas triangulaires et de souvenirs pyramidaux, transforment leur jardin en parking pour les touristes et leur grange en débit de boissons. Semir Osmanagić multiplie les allers et retours entre Houston et Visoko, et se lance dans une série de conférences et dédicaces de son livre, d'abord en Bosnie et dans les pays voisins, puis dans les ambassades et consulats de Bosnie à l'étranger. Parallèlement le nombre de "pyramides " autour de Visoko augmente, passant de deux à trois, puis cinq, et les fouilles entreprises par la Fondation s'étendent à de nouveaux sites: collines de Plješevica (dite "Pyramide de la Lune») et de Vratnica (considérée par Semir Osmanagić comme un tumulus artificiel), tunnels dits «KTK» et de Ravne (que Semir Osmanagić présente comme les entrées d'un réseau souterrain reliant les pyramides entre elles) $)^{14}$.

\section{B. La position des scientifiques}

Très vite cependant les premiers doutes apparaissent ; dès la fin de l'année 2005 en fait, lorsque les théories de Semir Osmanagić ont commencé à circuler en Bosnie, les archéologues et historiens locaux (par exemple Enver Imamović, de l'Université de Sarajevo, ou Zilka Kujundžić-Vejzagić, archéologue au Zemaljski Muzej de Sarajevo) ont exprimé leur scepticisme sur la possibilité d'une civilisation avancée dans les Balkans 
totalement inconnue des spécialistes, mais leur voix a été très généralement noyée dans la surexcitation ambiante. A l'étranger, des journalistes - Mark Rose ${ }^{15}$, Archaeology Magazine - et des archéologues - Anthony Harding ${ }^{16}$, Université d'Exeter et président de l'European Association of Archaeologists - ont pris la peine de se renseigner un peu plus sur Semir Osmanagić ; loin d'être archéologue, comme il est souvent présenté dans les premières dépêches, celui-ci peut même difficilement être considéré comme un archéologue amateur: il n'a jamais participé à aucun chantier de fouilles, et son expérience de l'archéologie se résume à des visites touristiques des pyramides d'Amérique centrale. Il n'a aucune publication scientifique à son actif, et la demidouzaine de livres qu'il a publiés se résument pour l'essentiel à des carnets de voyage ${ }^{17}$.

Plus gênant encore aux yeux des scientifiques, ces livres révèlent son adhésion sans réserve à quasiment toutes les idées pseudo-scientifiques, New Age, voire conspirationnistes, en vogue: ancêtres extraterrestres des Mayas, civilisations disparues de $\mathrm{Mu}$ et de l'Atlantide, complots maçonniques et des Illuminati, alignement galactique porteur de changements dramatiques en 2012 annoncé par le calendrier maya, bref un véritable pot-pourri des idées de José Argüelles, Erich von Dänicken, David Icke, Zecharia Sitchin, Richard Hoagland, ou encore Edgar Cayce. Son livre La Pyramide du Soleil de Bosnie replace sa découverte de Visoko dans ce contexte-là : la datation de 12000 ans pour les "pyramides " est avancée en référence au supposé cataclysme ayant détruit l'Atlantide, et, s'il n'y a aucune trace des mystérieux bâtisseurs des "pyramides ", on comprend que c'est parce qu'ils sont repartis - on se sait où.

11 L'argument selon lequel les idées personnelles discutables de Semir Osmanagić n'auraient pas d'importance tant que les fouilles sont correctement menées par des spécialistes ne tient pas longtemps: on apprend en effet rapidement que l'équipe internationale de scientifiques n'existe que sur le papier. Des archéologues mentionnés par Semir Osmanagić comme faisant partie du projet protestent, et demandent le retrait de leur nom du site de la Fondation. Ainsi, Grace Fegan, archéologue irlandaise, indique qu'elle avait contacté Semir Osmanagić suite à une annonce parue sur le site de l'Archaeological Institute of America, mais sans donner suite devant l'aspect douteux du projet; elle a ensuite découvert avec effarement son nom sur le site de la Fondation comme "Senior Archaeologist», avec une adresse email en @piramidasunca.ba, à laquelle elle n'avait aucun accès ${ }^{18}$. Des mésaventures semblables sont arrivées à l'archéologue australien Royce Richards, ainsi qu'au Canadien Chris Mundigler. Dans d'autres cas, le scientifique nommé sur le site de la Fondation a effectivement participé au projet, mais s'en est éloigné plus ou moins rapidement ou discrètement : ainsi, l'archéologue suédois Sead Pilav a pris ses distances avec Semir Osmanagić et sa Fondation dès le tout début du projet, considérant que celui-ci ressemblait plus à un «Big Brother Show » qu'à un chantier archéologique sérieux ${ }^{19}$.

12 De très nombreuses voix mettent en doute le fondement même du projet: les archéologues Anthony Harding ${ }^{20}$ ou Curtis Runnel ${ }^{21}$ évoquent la faible plausibilité de l'idée même de pyramides préhistoriques selon les connaissances actuelles sur le Paléolithique des Balkans, montrant en particulier l'impossibilité d'une entreprise de construction si gigantesque dans une société de chasseurs-cueilleurs, et l'absence de toute trace archéologique de la supposée civilisation des "pyramides"; les historiens (Enver Imamović, Dubravko Lovrenović) tirent de leur côté la sonnette d'alarme sur le risque réel d'atteinte au patrimoine archéologique antique ou médiéval: dans sa hâte 
d'exhumer les couches géologiques considérées comme les «murs de la pyramide », la Fondation prend le risque de détruire des sites réels, en particulier autour de la forteresse et cité médiévale de Visoki (une des capitales du royaume de Bosnie au Moyen Âge) située justement sur la colline de Visočica ${ }^{22}$. Les géologues Sejfudin Vrabac $^{23}$, Stjepan Čorić24, Paul Heinrich ${ }^{25}$, Robert $\mathrm{Schoch}^{26}$, sont quant à eux catégoriques: les dalles des "pyramides » révélées par les fouilles sont en fait des couches sédimentaires datées du Miocène et fracturées en réseaux plus ou moins orthogonaux de diaclases et de failles (Visočica se situe dans une zone tectoniquement encore active), et la forme des «pyramides » n'est que le résultat de l'action conjointe de facteurs tectoniques (soulèvement et basculement des couches sédimentaires émergées à la fin du Tertiaire) et de l'érosion, responsable des versants en $\mathrm{V}$ des cours d'eau.

\section{Poursuite des fouilles depuis 2006}

13 La cause semble donc entendue dès 2006, et la quasi-totalité des scientifiques se désintéresse de l'affaire les années suivantes. Pourtant, malgré ce désaveu de ce qu'il appelle la science "officielle", Semir Osmanagić persiste. Aujourd'hui, en 2011, non seulement les «pyramides» ne sont pas retombées aux oubliettes de la pseudohistoire, mais le projet semble relativement prospérer.

Le site archéologique de Visoko comprend maintenant tout un ensemble de structures :

- cinq "pyramides» ou collines autour de Visoko: du Soleil (Visočica), de la Lune (Plješevica), de l'Amour (Čemerac), de la Terre (Krstac), et du Dragon (Buci) ;

- un tunnel dit KTK, sur la rive gauche de la Bosna, dont l'entrée se situe sur le terrain de l'entreprise KTK (textiles et cuirs);

- un autre tunnel à proximité du village de Ravne, à environ trois kilomètres au Nord de Visočica;

- un «tumulus préhistorique » (colline de Toprakalja, près du village de Vratnica à quelques kilomètres au Nord-Est de Visoko);

- diverses structures (affleurements rocheux ou blocs de pierre isolés) disséminées dans la vallée de Visoko (Ginje, Dvor...).

À cela s'ajoute un autre élément, beaucoup plus distant : un ensemble de sphères de pierre (d'un diamètre allant de quelques dizaines de centimètres à plus d'un mètre), présentes dans plusieurs régions de Bosnie (et de la Serbie voisine), et dont la plus importante concentration se trouve dans le village de Zavidovići, à 50 kilomètres à vol d'oiseau au Nord de Visoko. Semir Osmanagić interprète ces sphères de pierre comme des artefacts préhistoriques, éléments d'un « réseau énergétique mondial » qu'il relie à $\mathrm{d}$ 'autres sphères présentes en particulier au Costa Rica ${ }^{27}$.

Dans cet ensemble, seules deux « pyramides », Visočica et Plješevica, les deux tunnels et le «tumulus» de Vratnica ont fait ou font l'objet de fouilles, non par choix de Semir Osmanagić, mais plutôt par manque de personnel. L'équipe permanente de la Fondation est en effet assez réduite; on y trouve, en dehors des membres du Conseil d'Administration qui ne participent pas aux travaux sur le terrain, très peu de permanents salariés. L'essentiel du travail est fait durant les mois d'été par des volontaires qui viennent à titre individuel ou, depuis 2010, dans le cadre d'un chantier international co-organisé avec la municipalité de Visoko ${ }^{28}$. Le recrutement de ces volontaires ne se fait pas par les filières classiques de l'archéologie, mais 
essentiellement par le bouche à oreille ou par le biais des forums et sites web consacrés à l'histoire "alternative" ou à diverses théories complotistes $^{29}$. La majorité des volontaires vient de Bosnie ou des pays voisins (Croatie, Slovénie), ou de la diaspora bosnienne, mais pas uniquement : en 2011, on peut trouver parmi eux par exemple un étudiant en géologie anglais, une femme policier australienne, un jeune professeur italien, un marchand de livres néerlandais, etc. Beaucoup sont très jeunes, encore étudiants - dont certains en archéologie ou géologie, ce qui permet à la Fondation de revendiquer le passage de « dizaines d'archéologues et géologues » sur le site ${ }^{30}$.

Une caractéristique de beaucoup de ces volontaires - du moins de ceux qui s'expriment sur les forums de discussion ou dans des interviews diffusés sur YouTube - est, à côté de leur conviction allant parfois jusqu'au quasi-fanatisme de la réalité des "pyramides", leur adhésion fréquente à diverses variantes des idées New Age ou ésotériques. Cette tendance se retrouve également dans l'entourage plus ou moins proche de Semir Osmanagić et au sein de sa Fondation. Le Directeur général de celle-ci, par exemple, est un certain Ahmed Bosnić : auteur de livres à succès consacrés à divers mystères (Atlantide, $\mathrm{Mu}$, extra-terrestres), il gère également un site de vente en ligne de produits ésotériques et magiques (horoscopes, talismans, potions variées) ${ }^{31}$. Un grand nombre de gourous, guérisseurs, voyants ou médiums a défilé à Visoko depuis $2006^{32}$, et Semir Osmanagić est fréquemment invité à l'étranger pour des conférences par diverses organisations ésotériques, voire sectaires, par exemple la secte Damanhur en Italie ${ }^{33}$.

\section{L'équipe scientifique}

Les scientifiques ne sont pas totalement absents de la Fondation, mais ils y sont très peu nombreux. De l'équipe impressionnante - et artificiellement gonflée - du début, ne reste en 2011 plus personne : le géophysicien Amer Smailbegović a rompu avec Semir Osmanagić en mai-juin 2006, les deux archéologues présentes en 2006 (la Serbe Silvana Čobanov et la Grecque Nancy Gallou) ont disparu à la fin de la saison, et la géologue en titre Nadija Nukić a claqué la porte en 2007, en même temps que le directeur du musée de Visoko (qui, bien que non archéologue lui-même, était en quelque sorte le garant scientifique du projet).

Depuis le départ de Nadija Nukić, la Fondation n'a plus jamais eu de géologue en titre l'origine naturelle des éléments présentés comme archéologiques par Semir Osmanagić étant probablement trop évidente aux yeux d'un géologue pour qu'il puisse de bonne foi adhérer à l'idée des "pyramides». Par contre, la Fondation a toujours réussi à engager un archéologue permanent en titre, comme l'y obligent ses statuts, même si parfois elle a eu du mal à le trouver. En 2007-2008, l'archéologue en titre était un jeune britannique, Andrew Lawler, qui, sans croire visiblement aux théories de Semir Osmanagić, a tenté d'organiser un peu le chantier et d'y imposer le minimum de méthode scientifique qui manquait jusque là. Suite à son départ de la Fondation en septembre 2008 (il reprochait entre autres à Semir Osmanagić d'avoir profondément remanié sans son accord un de ses rapports pour en ôter les analyses non conformes à la théorie des "pyramides $»^{34}$ ), Andrew Lawler a été remplacé par Mislav Hollos, un artiste sans compétence particulière en archéologie (titulaire d'un Master en Histoire de l'Art et Archéologie, le sujet de son mémoire portait sur la Comedia Dell'Arte dans l'oeuvre de Jacques Callot) ; puis, depuis l'été 2010, par une jeune archéologue italienne, 
Sara Acconci, titulaire d'un Master de l'Université de Milan ayant essentiellement travaillé en étruscologie.

Le point essentiel concernant les divers archéologues qui se sont succédé sur le chantier de Visoko est qu'on a presque toujours affaire à des professionnels très jeunes, souvent fraîchement diplômés et avec peu ou pas d'expérience. Quand on connaît la difficile condition de beaucoup de jeunes diplômés en archéologie en Europe, on comprend l'attrait que doit représenter la perspective d'un emploi permanent comportant la responsabilité d'un immense chantier. Malgré cela, tous ces archéologues sont entrés à un moment ou un autre en conflit avec Semir Osmanagić et ont fini par quitter le projet en essayant de faire oublier leur participation; seule Sara Acconci, qui semble avoir adopté les théories de son employeur, fait exception pour le moment.

Malgré la faiblesse de l'encadrement scientifique de ce que Semir Osmanagić présente comme "le plus grand projet archéologique du $\mathrm{xxl}^{\mathrm{e}}$ siècle ", le chantier s'est donc poursuivi, avec un soutien sans faille - ou presque - des autorités bosniennes et d'une bonne partie de l'opinion publique du pays depuis 2006. Les raisons de ce succès - tout relatif, puisque la Fondation n'a pas avancé d'un pouce dans la démonstration de l'existence des "pyramides", mais succès quand même, puisqu'il s'agit à ma connaissance du seul chantier pseudo-archéologique sur la planète financé sur fonds publics - sont très complexes.

\section{Les stratégies de Semir Osmanagić}

Le succès tient d'abord à l'efficacité des stratégies adoptées par Semir Osmanagić et la Fondation, à la fois dans l'utilisation d'éléments de la géologie et de l'archéologie locales, et dans le discours autour de ces éléments.

\section{A. Géologie de Visoko}

Une des racines de la réussite de l'affaire des "pyramides " tient à la géologie de la région de Visoko. La structure géologique de Visočica (un volet anticlinal avec une surface structurale constituant le versant Nord, les autres versants résultant de l'enfoncement des cours d'eau dans des vallées en V) n'a rien en soi de bien original. Mais le tour de force de Semir Osmanagić consiste en une assimilation des divers éléments naturels révélés par les fouilles à des éléments artificiels, en jouant sur des ressemblances superficielles mais relativement convaincantes pour le profane. Cette assimilation est réalisée par l'emploi systématique du vocabulaire de la construction et du génie civil en lieu et place du vocabulaire géologique : les couches de conglomérat inclinées sont les « murs » de la pyramide, les versants les «faces » de celle-ci; les blocs fracturés de conglomérat sont "découpés à angle droit", et lorsqu'une fracture ancienne est emplie d'un dépôt de calcite on a affaire à des blocs "cimentés"; les couches horizontales de grès de Plješevica qui offrent de beaux exemples de réseaux orthogonaux de fractures deviennent des «dallages" ou des "pavements". L'assimilation est également renforcée par l'utilisation de l'image : on ne compte plus sur le site de la Fondation les exemples de collages superposant une pyramide égyptienne ou méso-américaine à une des collines de Visoko; et les photographies qui circulent par milliers sur internet sont soigneusement choisies: seuls sont 
photographiés (et pas seulement par la Fondation : les touristes ont eux aussi tendance à choisir bien naturellement de photographier ce qui leur parait le plus frappant) les éléments qui ont le plus de ressemblance avec des constructions, les contre-exemples, eux (fractures irrégulières, variations de pendage d'un bloc à l'autre...), n'étant généralement pas montrés. La façon même de fouiller les "pyramides" vise au même but: renforcer les ressemblances superficielles avec des constructions antiques. On commence à dégager un angle de la pyramide (en fait le rebord d'une couche sédimentaire) sur quelques mètres, puis les fouilles s'arrêtent avant que l'apparente perfection de l'angle ne soit compromise, quelques mètres plus loin, par une fouille plus poussée qui révèlerait la présence d'autres couches. Parmi les milliers de fines couches horizontales alternant grès et marnes qui constituent la colline de Plješevica, on choisit soigneusement de dégager une couche de grès, celle qui ressemble le plus à un " pavement ", pour en faire une allée circulaire s'enroulant autour de la "pyramide » ou bien un élément d'une terrasse de pyramide à degrés.

Comment fabriquer une pyramide à degrés

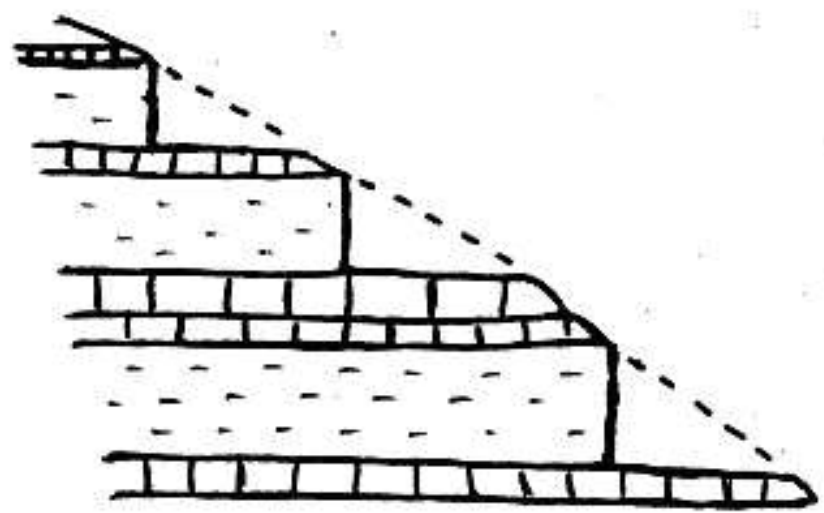

En pointillés, la pente originelle avant excavation

Source : « Géologie des « pyramides » de Bosnie », Le Site d'Irna, 24 novembre 2006 - http:// irna.lautre.net/Geologie-des-pyramides-de-Bosnie.html

D'après la géologue Nadija Nukić3 ${ }^{35}$, membre de l'équipe de la Fondation en 2006, il semble même que, sur les dizaines de sondages archéologiques pratiqués sur les deux collines, certains aient été volontairement refermés car révélant trop clairement la structure purement sédimentaire des "pyramides». S'il est aujourd'hui plus difficile, du fait de la présence des nombreux volontaires qui alimentent leur profil FaceBook des photographies prises pendant leur séjour, de cacher ainsi des sondages peu convaincants, l'explication de toute incohérence est cependant vite trouvée: si les fouilles de l'été 2011 n'ont pas réussi à révéler l'emplacement d'un nouvel angle de Visočica, c'est que cet angle a dû être détruit ${ }^{36}$.

Dans cette entreprise d'artificialisation du site, la Fondation a été aidée par la présence autour de Visoko de phénomènes bien connus des géologues, mais pas forcément du 
grand public, qu'il est donc relativement aisé de faire passer pour des éléments artificiels. On en prendra trois exemples :

- Les ripple-marks ou rides : ces ondulations régulières, connues de tous les vacanciers lorsqu'elles se forment sur le sable fin sous l'action des vagues sur une plage, mais fossilisées dans le cas qui nous occupe, sont assez fréquentes et bien développées dans certaines couches de Plješevica. Elles trahissent les conditions du dépôt de ces couches en eau peu profonde dans le lac qui emplissait la totalité du bassin de Zenica-Sarajevo au Miocène, mais la Fondation n'a guère eu de mal à les faire passer pour des décorations, des sculptures faites volontairement sur les dalles.

- Le conglomérat qui surmonte les grès et marnes sur Visočica, formé de blocs plus ou moins arrondis cimentés dans une matrice calcaire, lui aussi déposé dans le lac Miocène pendant une période de surrection active des massifs bordant le bassin de ZenicaSarajevo, offre une ressemblance suffisante avec un béton artificiel pour que la Fondation n'ait eu semble-t-il guère de difficulté à le faire passer pour tel.

- Les sphères de pierre, dont Semir Osmanagić veut faire des artefacts rattachés à la civilisation des " pyramides ", sont des concrétions diagénétiques géantes développées à l'intérieur de bancs de grès massifs. On connaît d'autres exemples dans le monde de ces concrétions métriques (Saint-André-de-Rosans en France, les Moeraki Boulders en Australie, pour ne citer qu'elles), mais Semir Osmanagić joue sur la ressemblance de ces concrétions avec d'autres sphères géantes qui, elles, sont bien des artefacts : celles du Costa Rica.

\section{B. Un patrimoine archéologique réel}

Un autre élément utilisé par Semir Osmanagić pour renforcer sa théorie est la richesse archéologique réelle de la région. La présence continue de l'homme est attestée autour de Visoko au moins depuis le Néolithique: sites néolithiques et chalcolithiques nombreux, dont le plus important est celui d'Okolište ${ }^{37}$, fouillé depuis 2002 par une équipe germano-bosnienne dirigée par Johannes Müller (Université de Kiel) et Zilka Kujundžić-Vejzagić (Zemaljski Muzej de Sarajevo) ; fréquentes traces d'aménagements défensifs illyriens puis romains; très nombreux sites médiévaux : forteresse et cité médiévale de Visoki, église et cimetière des rois de Bosnie à Mile/Arnautovići ${ }^{38}$.

Face à cette richesse archéologique qu'elle est accusée de menacer, la Fondation de Semir Osmanagić hésite entre plusieurs attitudes. Parfois c'est une attitude de négation, si la présence d'un site attesté est susceptible de gêner sa propre activité : ainsi, la taille et l'importance de la forteresse de Visoki ${ }^{39}$ ont longtemps été minorées par Semir Osmanagić, qui affirmait qu'il n'en restait pas « pierre sur pierre » depuis sa destruction lors de la conquête ottomane ${ }^{40}$, avant que les travaux de fouilles et restauration entamés en 2008 ne lui apportent un démenti sous forme de vestiges impressionnants ${ }^{41}$.

Plus souvent, au contraire, la Fondation se livre à une véritable entreprise de détournement de ce patrimoine archéologique, lorsque certains éléments sont susceptibles d'être intégrés à la théorie des "pyramides». C'est le cas des stećci, emblématiques du patrimoine bosnien; ces dizaines de milliers de monuments funéraires médiévaux, présents en Bosnie-Herzégovine et dans quelques pays voisins, offrent souvent une iconographie très originale, et ont donné lieu à de riches études depuis le $\mathrm{xix}^{\mathrm{e}}$ siècle $^{42}$. La présence d'une nécropole médiévale et de stećci est attestée 
sur le petit plateau qui s'étend immédiatement à l'Ouest de Visočica, mais les quelques stećci encore visibles sont présentés par Semir Osmanagić comme « des blocs tombés de la pyramide ", et au moins un squelette déterré lors des premiers sondages a purement et simplement disparu sans pouvoir être datée ${ }^{43}$. De nombreux textes, publiés sur le site de la Fondation ou sur des sites partenaires, émanent d'auteurs qui tentent soit de repousser l'âge des stećci à un lointain passé pour en faire des éléments de la civilisation des «pyramides» (ainsi Semir Osmanagić dans son livre Bosanska Piramida Sunca présente-t-il un stećak comme un « autel astronomico-énergétique » préhistorique), soit d'en faire des témoins de l'existence même des "pyramides": la forme de certains stećci, avec un couvercle triangulaire, serait inspirée par les "pyramides » de Visoko, leur décoration originale (spirales, rosaces) serait constituée de symboles mystiques hérités de la civilisation des "pyramides ${ }^{44}$, ou renverrait à un mode d'emploi de «pyramides » supposées être de gigantesques machines climatiques ${ }^{45}$.

Les sites préhistoriques connus ne sont pas à l'abri de ces détournements. Le site d'Okolište par exemple, un des plus grands tells néolithiques connus dans les Balkans avec une population probable de 3000 personnes, ce qui en fait un site clé pour la question de la transition vers des sociétés urbaines complexes ${ }^{46}$, est utilisé par Semir Osmanagić ou les tenants des "pyramides", qui mentionnent régulièrement «les dizaines de milliers d'artefacts trouvés par les archéologues allemands ", en espérant renforcer l'idée d'une civilisation des "bâtisseurs des pyramides " à Visoko, oubliant toutefois de préciser que ces milliers d'artefacts n'ont pas été trouvés près des " pyramides» et n'ont aucun rapport avec elles. Un de ces artefacts en particulier a suscité beaucoup d'intérêt: daté par les archéologues du début du Chalcolithique, cet artefact de forme pyramidale est utilisé par les partisans des "pyramides» comme preuve de leur existence ${ }^{47}$, alors que son sommet brisé montre à l'évidence qu'il ne s'agit pas d'un objet complet, mais du pied ou support d'autre chose, probablement un encensoir ou un autel miniature ${ }^{48}$.

31 Un autre cas possible de récupération d'un site réel est peut-être le tunnel de Ravne. De nombreux éléments de ce tunnel (morphologie, présence d'un système de drainage, galeries abandonnées comblées de stériles et murées ${ }^{49}$ ) évoquent une mine (non datable actuellement) dans un gisement alluvionnaire ancien, plutôt qu'un réseau souterrain de communication avec des «pyramides » dont la plus proche est à trois kilomètres, de l'autre côté d'un vallon.

32 Enfin, il arrive que Semir Osmanagić se pose en défenseur du patrimoine archéologique bosnien. Ainsi, lorsqu'est annoncé le déblocage par les autorités fédérales de crédits pour la fouille et la restauration de la forteresse et ville médiévale de Visoki, la Fondation propose pour cette forteresse (jusque là considérée comme inexistante, voir plus haut) son propre projet: sur 51 pages, celui-ci n'en consacre réellement qu'une seule à la ville de Visoki, et les actions proposées tiennent sur 6 lignes $^{50}$.

On a donc dans le cas des " pyramides » de Visoko affaire à une véritable chimère ${ }^{51}$, une création fabuleuse mêlant éléments géologiques et éléments archéologiques. Cette chimère géologico-archéologique, Semir Osmanagić la présente au monde en usant en permanence de deux discours antinomiques, selon son public: le discours scientifique (ou du moins son apparence), et le discours anti-élitiste et anti-intellectuel. 


\section{L'apparence de la science}

34 Le premier, le discours scientifique ou supposé tel, est réservé essentiellement aux hommes politiques ${ }^{52}$ et à la presse, particulièrement étrangère. Ce discours scientifique tient en fait plus de l'incantation que d'autre chose. Un bon exemple en est le seul rapport de fouilles publié par la Fondation, et adressé à toutes les administrations du pays $^{53}$. Ce rapport liste sur des paragraphes entiers les analyses qui pourraient être pratiquées - mais qui ne l'ont jamais été, noyant le lecteur sous une avalanche de mots impressionnants ( analyses minéralo-pétrographiques, sédimentologiques, aux rayons $\mathrm{X}$, thermiques différentielles, thermogravimétriques, ensuite analyses de l'âge par la méthode des isotopes radioactifs du carbone, de l'oxygène, du potassium-argon, du rubidium-strontium, puis micro- et macro- analyses paléontologiques, analyses chimiques, géochimiques et autres analyses, à côté d'analyses physico-mécaniques et géomécaniques...»); ou bien fournit des documents bruts (radargrammes, mesures sismométriques de réfraction) sans aucune analyse ou interprétation, donc incompréhensibles au commun des mortels, dont font à coup sûr partie la plupart des destinataires du rapport. En revanche, les éléments essentiels d'un rapport de fouilles sont absents : aucun plan des fouilles, aucun relevé, par exemple. L'ensemble n'est donc ni un texte scientifique, ni un texte de vulgarisation, et semble n'avoir qu'un but : créer l'illusion de la scientificité pour des non scientifiques.

Cette obsession de l'apparence de la science est manifeste chez Semir Osmanagić. Elle se traduit, comme on l'a vu plus haut, par d'incessantes annonces de participation de scientifiques dont la plupart ne mettront jamais les pieds sur le chantier; mais aussi par une véritable course aux titres de Semir Osmanagić lui-même, probablement gêné par son absence de statut scientifique. C'est d'abord la soutenance d'une thèse de sociologie sur les Mayas ${ }^{54}$ - devant un jury de l'Université de Sarajevo dont aucun membre n'est archéologue ni spécialiste des Mayas, thèse dont la bibliographie, où se côtoient guides touristiques et références à Auguste Le Plongeon ou José Argüelles, ferait hausser les sourcils à plus d'un mayaniste, mais qui lui permet d'obtenir le titre de Docteur. C'est ensuite l'adhésion à divers organismes plus ou moins scientifiques, en particulier à l'Académie des Sciences Naturelles de Russie (RAEN). Présentée comme "prestigieuse " par la Fondation, cette Académie n'a pas grand chose à voir avec l'Académie des Sciences de Russie (RAN), et n'est semble-t-il qu'une des nombreuses pseudo-académies privées qui ont éclos en Russie après la chute du communisme, celleci étant de plus particulièrement liée à la théosophie et aux cercles ésotériques russes. A ces titres de Docteur et Académicien, Semir Osmanagić ajoute de plus celui de Professeur d'Anthropologie, ayant réussi à obtenir la responsabilité d'un cours d'été sur les "sites mégalithiques bosniens" pour les étudiants d'une université privée de Bosnie, l'American University of Bosnia and Herzegovina (AUBiH).

\section{Conférences scientifiques et analyses}

Une autre manifestation de cette imitation formelle de la science est l'organisation, par la Fondation, de conférences internationales consacrées aux « pyramides " à Sarajevo, la première ayant eu lieu en septembre $2008^{55}$ et la deuxième en septembre $2011^{56}$. L'organisation de ces manifestations reprend dans les moindres détails le déroulement des rendez-vous scientifiques: appels à contributions, excursions pré- ou postconférence, badges, publication des Actes de la conférence, etc. Les participants à la 
première conférence de $2008^{57}$ pourraient être classés en trois catégories : dans la première, les amis de Semir Osmanagić, ceux qui partagent l'essentiel de ses idées New Age : le journaliste Philip Coppens (spécialiste de Rennes-le-Château, du Graal, et auteur d'un livre intitulé The New Pyramid Age), le glozelien et « hiérolinguiste " ${ }^{58}$ Paulo Stekel, les « chercheurs indépendants » en histoire alternative Višnja Dobrić et Gabriela Lukacs, etc.

Dans la deuxième catégorie, on trouve des scientifiques légitimes, qui ont une carrière tout à fait orthodoxe, mais qui semblent appuyer largement les théories de Semir Osmanagić sur les "pyramides » de Bosnie, et donc leur conférer une certaine validité scientifique. Parmi ceux-ci, les seuls à avoir publié quelque chose sont le minéralogiste égyptien Aly Barakat, et l'égyptologue, égyptien également, Nabil Swelim. Le Dr Barakat a publié en 2007 sur le site de la Fondation un court rapport de 4 pages ${ }^{59}$, où il semble pencher vers une origine naturelle de la colline de Visočica, mais en affirmant cependant, sans véritable argumentation scientifique, une intervention humaine qui aurait eu pour effet «d'adapter la forme de la colline pour la transformer en pyramide». Le Dr Swelim, lui, a publié en 2008 un texte beaucoup plus long ${ }^{60}$ dans lequel il affirme l'existence des pyramides en Bosnie, mais en donnant à ce mot de "pyramides» un sens purement géométrique, sans vraiment se prononcer sur l'artificialité desdites pyramides; et il propose la création d'une nouvelle catégorie de pyramides, celle des « collines pyramidales bosniennes ». Le ton général du texte, ainsi que l'emploi d'un vocabulaire renvoyant clairement à celui de la construction, laissent penser que l'auteur soutient la thèse de pyramides artificielles; cependant, un autre texte ${ }^{61}$, publié cette fois uniquement sur le site personnel du Dr Swelim en 2010, semble laisser entrevoir la position inverse: si l'auteur maintient l'appellation de " pyramides » - au sens géométrique - pour les collines de Visoko, il constate qu'aucun élément archéologique ne permet d'affirmer une origine artificielle. Il est à noter qu'aucun de ces deux auteurs n'a publié quoi que ce soit sur les «pyramides » de Bosnie dans une revue scientifique (le Dr Swelim ayant même, semble-t-il, décliné l'offre qui lui avait été faite par Blagoje Govedarica, de l'Académie des Sciences et Arts de BosnieHerzégovine, de publier dans les Annales du Centre d'Études Balkaniques, si l'on en croit la correspondance entre le Dr Govedarica et le Dr Swelim publiée sur le site de la Fondation ${ }^{62}$ ); aucun non plus, ni d'ailleurs aucun de leurs collègues égyptiens, n'est présent à la deuxième conférence de 2011, sans qu'on puisse savoir si cette absence tient aux évènements ayant affecté l'Égypte en cette année 2011, ou à autre chose.

Enfin, la troisième catégorie de participants à la conférence de 2008 comprenait des scientifiques visiblement fourvoyés, croyant participer à une vraie conférence scientifique sur les pyramides. Le sujet de leurs interventions n'avait rien à voir avec les «pyramides» de Bosnie, mais bien avec des recherches archéologiques classiques sur les mausolées de la dynastie des Han occidentaux, la pyramide de Saqqarah ou la bibliothèque antique d'Alexandrie. Aucun de ces scientifiques ne s'est exprimé sur le sujet des «pyramides » de Bosnie, ni pendant ni après la conférence, et aucun n'a souhaité visiblement renouveler sa participation à la conférence de 2011.

Cette dernière, à la différence de la précédente de 2008, ne semble guère avoir eu de succès parmi les scientifiques en général : la quasi-totalité des participants sont des " chercheurs indépendants ", amateurs soit de mystères archéologiques (le journaliste Philip Coppens, David Hatcher Childress, Klaus Dona), soit de physique «alternative " (Slobodan Mizdrak, Gaibija Ćatić, Goran Marjanović). On y trouve même un « Maître ès 
Sciences ", Domagoj Nikolić, qui enquête sur les "pyramides » à l'aide de son épouse médium ; la Fondation affiche sur son site la retranscription d'une session au cours de laquelle, entre autres, l'origine extra-terrestre des " pyramides » est affirmée ${ }^{63}$. Le seul participant à cette deuxième conférence qui peut prétendre à une dimension académique est le professeur Paolo Debertolis: odontologue, celui-ci enseigne l'archéologie dentaire à l'Université de Trieste en Italie; mais il est aussi un grand amateur de pseudo-archéologie et autres mystères, ainsi que de fin du monde en 2012, à quoi il consacre un site intitulé Salviamoci nel $2012^{64}$, et participe régulièrement à plusieurs forums italiens aux noms évocateurs : «nibiru2012.it », « ufoforum.it ». Le professeur Debertolis a formé fin 2010 un groupe de recherche (SB Research Group ${ }^{65}$ ) comprenant essentiellement, en sus de lui-même, une architecte de l'Institut Polytechnique de Milan, Lucia Krasovec Lucas, et la jeune archéologue Sara Acconci, déjà évoquée, qui est en même temps l'archéologue en titre de la Fondation. Cette équipe plus ou moins parallèle à celle de la Fondation a développé au cours de l'année 2011 ses activités dans trois directions principales :

- Recherches sur les stećci, en collaboration avec le Musée de Visoko ; ces recherches, d'abord très influencées par les théories de Semir Osmanagić et de quelques autres sur les stéci comme témoins d'une mystérieuse civilisation liée aux "pyramides", semblent se réorienter à partir de la fin de l'été 2011 vers une étude plus classique d'anthropologie sur les inhumations du début de la période moderne liées aux stećci.

- Recherches sur diverses formes d'énergie supposée émise par les "pyramides" (ultrasons, rayonnements électromagnétiques), recherches largement inspirées de celles menées dans les années 1970 sur des cercles mégalithiques au Royaume-Uni (Dragon Project par exemple sur les Rollright Stones ${ }^{66}$ ).

- Enfin, recherches sur l'architecture du tunnel de Ravne et les artefacts supposés qu'il contient.

Il semble cependant, à la lecture du seul forum d'archéologie sur lequel le professeur Debertolis intervient ${ }^{67}$, que celui-ci et son groupe subissent déception sur déception, découvrant en particulier que les résultats des analyses réalisées par la Fondation les années précédentes, sur lesquels ils s'étaient appuyés pour construire diverses théories, se révèlent assez peu fondés.

41 Le recours aux analyses, réelles ou supposées, est un autre exemple de la façon dont la Fondation de Semir Osmanagić cherche à donner une allure scientifique au projet. Cet aspect devrait être particulièrement développé dans la thèse de Tera Pruitt, doctorante à l'Université de Cambridge, intitulée Authority and the Production of Knowledge in Archaeology ${ }^{68}$. Le site de la Fondation multiplie les annonces d'analyses, mais la plupart de ces supposées analyses n'ont jamais fait l'objet d'une publication sous quelque forme que ce soit. Ainsi, des échantillons de conglomérat, prélevés on ne sait ni où, ni dans quelles conditions, auraient été soumis à l'Institut Polytechnique de Turin, qui aurait confirmé la nature artificielle du matériau ${ }^{69}$. Une photocopie - de très mauvaise qualité et donc illisible - de ce qui est présenté comme trois pages du rapport de cet Institut, a été publiée dans un magazine New Age italien, mais le rapport lui-même est introuvable et n'a jamais été présenté par la Fondation. D'autres rapports d'analyses ont eux bien été publiés, mais sont utilisés comme confirmation d'éléments qui n'ont en fait pas grand-chose à voir avec leur contenu. Ainsi, toujours sur la question de l'origine prétendument artificielle du conglomérat de Visočica, la Fondation annonce comme élément en faveur de cette thèse des analyses pratiquées par l'Institut de Génie 
Civil de Tuzla ${ }^{70}$ et le professeur Muhamed Pašić de l'Université de Zenica ${ }^{71}$, qui ne sont en fait que des mesures de la résistance à la compression réalisées au scléromètre. De telles mesures (généralement pratiquées pour évaluer la qualité d'un béton) ne peuvent donner d'indication que sur la solidité d'un matériau, mais en aucun cas sur sa composition ni son origine. Les datations au carbone 14 réalisées par la Fondation (une demi-douzaine en cinq ans) semblent tout aussi discutables, soit du fait des conditions de prélèvement, soit du fait de l'assimilation abusive d'un matériau à du matériau organique datable (datation par exemple à 32000 ans d'un matériau qui est très probablement du charbon d'âge Miocène rajeuni suite à une contamination par du carbone organique récent ${ }^{72}$ ), soit encore du fait d'une interprétation, là aussi abusive, de la nature et donc de la signification du matériau daté (filon de calcite présenté comme une stalagmite). Ces datations très discutables et ces analyses non concluantes sont utilisées par la Fondation comme étendard scientifique, dans une forme inédite de « culte du cargo $»^{73}$.

\section{E. Une science « populaire»}

Mais, à côté de cette facette apparemment scientifique, le discours de Semir Osmanagić offre aussi d'autres facettes, bien différentes. Il se présente facilement comme le héraut d'une science " populaire » opposée à une science « officielle " sclérosée. Ressortissent à cette stratégie ses nombreuses références à divers scientifiques "maudits " et persécutés par la "science officielle » de leur époque : Galilée, Tesla, etc. Ces références sont encore plus nombreuses chez ses admirateurs (presse populaire bosnienne, forums) qui lui construisent une image de génie incompris. Les allusions méprisantes aux «scientifiques en fauteuil » (foteljaši) éloignés de la réalité du terrain, aux élites scientifiques incapables de remettre en question leurs certitudes, sont fréquentes sous la plume de Semir Osmanagić et de ses partisans. Si ses thèses ne sont pas reconnues par la science officielle, ce n'est pas dû à l'absence de validité scientifique de son argumentation ou de ses analyses; c'est que les scientifiques officiels sont murés dans leurs certitudes, qu'ils refusent d'ouvrir les yeux sur le "nouveau paradigme" développé par les «chercheurs indépendants $»^{74}$. L'opposition des scientifiques au projet de Semir Osmanagić ne peut être qu'intéressée, soit qu'ils agissent par intérêt personnel : les chercheurs et professeurs auraient peur pour leur carrière menacée par la révélation que tout ce qu'ils enseignent est faux ; soit qu'ils aient à cœur un intérêt national : l'opposition des scientifiques, tant bosniens qu'étrangers, aux "pyramides » de Bosnie est ainsi présentée comme le résultat d'un complot au cœur duquel on trouverait Zahi Hawass, ancien secrétaire général du Conseil Suprême des Antiquités égyptiennes, et qui ne s'expliquerait que par la peur des Égyptiens de perdre au profit de la Bosnie une grosse part de la manne touristique liée aux pyramides ${ }^{75}$.

La science que prétend pratiquer Semir Osmanagić n'est donc pas une science réservée à l'élite (l'introduction d'un de ses ouvrages, od masona do mentalnih projekcija - [Des francs maçons aux projections mentales], évoque les «élites cachées » qui domineraient le monde ${ }^{76}$ ), mais une science pour le peuple : «I am not interested in the approval of elite scientists. This project is for the people ${ }^{77}$. Son choix d'offrir au monde une ressemblance étudiée avec l'icône populaire Indiana Jones n'est pas anodin : Indiana Jones est à la fois le symbole de l'archéologie mystérieuse, l'antithèse du "scientifique en fauteuil », et la représentation la plus populaire de l'archéologue pour le grand public. Toute la stratégie de communication de la Fondation est basée sur cette 
idée d'une science ouverte, transparente, immédiatement accessible au public sans le filtre des scientifiques; d'où l'abondance par exemple de témoignages de visiteurs publiés sur le site, sur le thème de l'évidence : il suffit de venir à Visoko pour « voir de ses propres yeux ", et pour être convaincu que «la nature ne peut pas faire ça »; d'où l'abondance également des images (photographies de blocs géométriques et de pavements, collages et montages suggestifs) remplaçant le texte et tenant lieu d'analyse scientifique. La communication de la Fondation n'est jamais scientifique (à ma connaissance, ni Semir Osmanagić, ni aucun des scientifiques qui ont collaboré plus ou moins brièvement au projet, n'a jamais tenté de soumettre un article à une revue scientifique); c'est une communication qui utilise des techniques de relations publiques : beaucoup de vidéos, communiqués choc dans la presse populaire, répétition ad infinitum de quelques idées simples, insistance sur des coïncidences présentées comme mystérieuses ${ }^{78}$, et appel du pied à diverses croyances ou thèmes populaires dans une certaine presse (effets thaumaturgiques des pyramides, recours à des sourciers, devins, et manipulateurs de gadgets pseudo-scientifiques variés ${ }^{79}$ ).

Il est bien évident que cette stratégie de communication ne renforce pas la crédibilité de la Fondation et de Semir Osmanagić aux yeux des scientifiques, et on peut se demander pourquoi ce dernier essaie ainsi, parfois acrobatiquement, de jouer sur les deux tableaux, science et pseudo-culture populaire. La réponse qui se dégage de plus en plus clairement, au fur et à mesure que les années passent, est que son projet n'a jamais été un projet scientifique: la science - peu, et mal - pratiquée par la Fondation, ressortit plutôt à une tentative de légitimation d'un projet guère plus fondé scientifiquement que par exemple le feu « Mystery Park » d'Erich von Däniken ${ }^{80}$.

\section{Un contexte favorable}

Pourtant ce projet, qui n'a de scientifique qu'une apparence très limitée, a rencontré en Bosnie - du moins à ses débuts, l'euphorie étant progressivement retombée par la suite - un succès extraordinaire. C'est d'abord un succès dans l'opinion, facilité par la très grande mansuétude dont ont fait preuve les médias locaux pour Semir Osmanagić et ses théories. La presse locale, audiovisuelle comme écrite, a fait peu de place aux opinions sceptiques, et s'est illustrée par une reprise systématique et textuelle des communiqués de la Fondation. Les affirmations les plus osées de Semir Osmanagić sont présentées comme des vérités scientifiques, sans effort aucun de recoupement et de vérification. Lors des conférences de presse de la Fondation, les journalistes présents ne posent aucune question dérangeante ${ }^{81}$. Les seuls médias à avoir de temps en temps donné la parole aux opposants du projet sont l'hebdomadaire de Sarajevo BHDani et, plus rarement, le quotidien Oslobođenje. Ce soutien global de la presse s'explique en partie par les relations personnelles de Semir Osmanagić au sein des milieux politiques et médiatiques - sa compagne travaille au sein de la FENA, l'agence de presse bosnienne, et son père, Muris Osmanagić, homme politique et ancien ministre de Yougoslavie, semble avoir gardé un réseau de relations étendu - mais pas seulement : la campagne de presse commencée par la Fondation au printemps 2006 n'a pu réussir que grâce au contexte particulier de la Bosnie d'après-guerre. 


\section{A. Une opportunité touristique}

Dans ce pays qui est l'un des plus pauvres d'Europe (avant-dernier selon le PIB/hab), et qui peine à se reconstruire après un conflit meurtrier marqué par un retour à une barbarie inouïe depuis 1945 sur ce continent, le projet de Semir Osmanagić est apparu à beaucoup de Bosniens comme une chance, comme un juste retour de l'histoire. Une chance d'abord économique: l'occasion de susciter des flux touristiques plus importants dans un pays qui ambitionne de développer ce secteur, en s'appuyant sur la qualité de son patrimoine naturel et la richesse de son patrimoine historique. La ruée annoncée des touristes vers Visoko, et plus globalement vers la Bosnie tout entière, est un argument fréquemment avancé par Semir Osmanagić parmi les raisons de soutenir son projet; il s'étend volontiers sur la comparaison avec l'Égypte, qui encaisserait chaque année 15 milliards de dollars de recettes touristiques grâce à ses pyramides, et pronostique un détournement vers la Bosnie d'une part appréciable de ces recettes dans le futur. Cet argument, d'autant plus fort que Semir Osmanagić est lui-même un homme d'affaires prospère, est facilement entendu, tant des hommes politiques (selon Haris Silajdžić, membre de la présidence collégiale de Bosnie-Herzégovine en 2006: "Let them dig and we'll see what they find. Besides, it's good for business ${ }^{82}$ ) que de la population locale : avec un taux de chômage dépassant les $40 \%$, les habitants de Visoko ont cru en un boom touristique fabuleux: les prix des terrains se sont envolés, beaucoup d'habitants se sont lancés dans de petites activités plus ou moins improvisées : buvettes, commerces de souvenirs, guides, gardiens de parking, la liste n'est pas exhaustive ${ }^{83}$. Six années après l'euphorie des débuts, les espoirs de décollage économique de Visoko ont semble-t-il été quelque peu déçus; le nombre exact de touristes est difficile à estimer, les chiffres avancés par la Fondation (400 000 visiteurs par exemple pour 2006) semblant très fantaisistes; l'office de tourisme cantonal n'a apparemment pas enregistré de hausse significative du nombre de nuitées sur le secteur, et les commerçants de Visoko se plaignent de retombées limitées, les touristes ne faisant généralement que passer sans séjourner sur place. Mais, malgré tout, le site des "pyramides » attire tant bien que mal un flux plus ou moins régulier de visiteurs (essentiellement des voyages organisés par quelques agences, dont TA Respect, agence officielle de la Fondation; des voyages scolaires; et plus occasionnellement des touristes indépendants) qui font vivoter quelques petits commerces. Malgré cette relative déception, Semir Osmanagić (parfois surnommé "Oncle Sam») apparaît toujours comme l'incarnation du rêve américain, un expatrié ayant réussi, un entrepreneur à succès, et donc symbole d'espoir en un avenir meilleur.

\section{B. Le poids des nationalismes}

Un autre élément, plus important sans doute encore, du soutien populaire aux théories de Semir Osmanagić est probablement à chercher du côté de l'histoire récente du pays et du conflit des nationalismes. Là encore, le discours tant de Semir Osmanagić que de ses partisans est très ambivalent. Le projet est souvent présenté par eux comme un projet fédérateur, unificateur : les « pyramides » n'appartiennent à aucune nation, elles sont l'occasion pour tous les peuples constituant la Bosnie-Herzégovine de travailler ensemble pour un monde meilleur. Cependant, c'est dans la part bosniaque (musulmane) de la population de Bosnie-Herzégovine que le projet a rencontré le plus d'adhésion ; ainsi, une enquête réalisée auprès d'étudiants des communautés bosniaque 
et croate de Mostar $^{84}$ montre un lien très net entre appartenance à la communauté ethnique bosniaque et croyance en l'existence et en l'importance des "pyramides ». Celles-ci sont apparues souvent comme une revanche des Bosniaques sur l'histoire, quasiment comme une compensation aux souffrances de la guerre ${ }^{85}$. Cet aspect est particulièrement présent chez les Bosniaques de la diaspora, pour lesquels le traumatisme de l'exil s'ajoute à celui du conflit, et qui forment le gros des soutiens et relais du projet à l'étranger. Un mémoire de master de Lejla Tricic ${ }^{86}$ développe abondamment ce thème, montrant comment «la fiction nationale des pyramides est créée pour surmonter la douleur et la souffrance, et pour guérir » (" pyramid fictional national narrative is created to overcome the pain and suffering, and to heal »).

Dans les faits, les adversaires du projet sont souvent assimilés à des traîtres ou des antimusulmans; s'il advient qu'ils soient croates (comme l'archéologue Zilka KujundžićVejzagić) ou serbes (comme le ministre de la Culture Gavrilo Grahovac), leur appartenance ethnique est mise en avant pour expliquer leur opposition. L'auteur même du présent texte, bien que française, s'est vue accusée sur un forum par des partisans des "pyramides" d'être "une ancienne personnalité politique serbe" cherchant à "continuer la guerre contre les Musulmans de Bosnie " par d'autres moyens ${ }^{87}$. Semir Osmanagić lui-même, s'il n'a jamais officiellement présenté les " pyramides " comme "bosniaques " plutôt que "bosniennes ", ne fait pas mystère dans ses ouvrages de ses convictions : les Bosniaques actuels seraient les descendants d'un peuple pré-Illyrien ${ }^{88}$, bâtisseur des "pyramides », et bénéficieraient donc d'une généalogie nettement plus prestigieuse que les Slaves, derniers arrivés dans la péninsule balkanique ${ }^{89}$. À ce titre, le projet des "pyramides" de Bosnie peut se rattacher aux diverses tentatives apparues dans les Balkans ces vingt dernières années d'ethnicisation de l'histoire et de l'archéologie (voir par exemple le recueil Historijski mitovi na Balkanu ${ }^{90}$ ou les Actes de la 14ème conférence annuelle de l'Association des Archéologues Européens à Malte en $2008^{91}$ ): une archéologie nationale fantasmatique, visant à faire de la Bosnie le « berceau de la civilisation européenne » (voire mondiale, la «pyramide du Soleil » étant «la mère de toutes les pyramides»), et à doter les Bosniaques d'aujourd'hui d'ancêtres prestigieux et d'une identité incomparablement plus ancienne que celle de leurs voisins et rivaux slaves.

\section{La faiblesse des institutions}

À ces éléments qui peuvent permettre d'expliquer au moins partiellement le succès de l'entreprise de Semir Osmanagić en Bosnie, il faut ajouter un autre facteur favorable : la relative faiblesse des institutions issues des Accords de Dayton, et le contexte politique qu'elles entraînent. La structure hyper-fédérale de la Bosnie-Herzégovine (deux entités, dont une Fédération croato-bosniaque elle-même divisée en dix cantons dotés de pouvoirs décentralisés) explique en partie (en sus des relations politiques de son père) la facilité avec laquelle Semir Osmanagić a pu obtenir autorisations de fouilles et subventions. Les autorités fédérales (de la Fédération croato-bosniaque) ont offert au projet un soutien mitigé : de nombreux membres du gouvernement fédéral ont encouragé Semir Osmanagić, visité les fouilles de Visoko (particulièrement au début, au moment où l'euphorie suscitée par le projet était à son comble; ces visites se sont ensuite quelque peu espacées au fur et à mesure que la presse se faisait moins présente sur le site). Mais la Fondation n'a pas réussi à obtenir du gouvernement fédéral le financement massif espéré, essentiellement du fait de l'opposition du ministre de la 
Culture et des Sports, Gavrilo Grahovac. Celui-ci, s'appuyant sur les avis sans appel rendus par diverses institutions (Commission pour la Protection des Monuments Nationaux $^{92}$, Zemaljski Muzej de Sarajevo ${ }^{93}$, Conseil fédéral pour la Géologie ${ }^{94}$, Académie des Sciences et Arts de Bosnie-Herzégovine ${ }^{95}$ ), a très tôt exprimé une opinion négative sur le projet, et a réussi à limiter l'engagement financier fédéral. Mais cette position fédérale n'a pas empêché les collectivités locales (cantons de Zenica-Doboj et de Sarajevo, municipalité de Visoko) de financer la Fondation, soit directement, soit par l'intermédiaire d'investissements structurels (financement de la route menant aux " pyramides »).

50 La même incohérence entre position fédérale et positions locales se retrouve en ce qui concerne les autorisations de fouilles. Au niveau fédéral, le ministre de la Culture considère que la Fondation n'offre pas les garanties scientifiques suffisantes pour pouvoir être considérée comme habilitée à procéder à des fouilles archéologiques ${ }^{96}$; pis, même, la Commission pour la Protection des Monuments nationaux estime que l'amateurisme du travail de la Fondation met en danger des sites archéologiques réels. C'est ce qui explique que Semir Osmanagić n'a jamais pu obtenir l'autorisation qu'il souhaitait de pratiquer des fouilles au sommet de Visočica, à proximité de la forteresse de Visoki classée Monument National ; et que les fouilles commencées en 2006 sur les pentes supérieures de la colline ont dû être abandonnées en 2007, suite à l'extension de la protection sur une zone beaucoup plus importante décidée par la Commission ${ }^{97}$. Par contre, ailleurs, en l'absence de monument classé, les autorisations de fouilles sont du ressort des autorités cantonales, qui les ont accordées année après année sur les collines de Plješevica et de Vratnica, malgré les mises en garde de la Commission et du ministre Grahovac, et l'absence de documentation scientifique sérieuse produite par la Fondation. À cela s'ajoutent fréquemment des rivalités entre échelons de décision différents, ainsi entre le Musée local de Visoko, qui a soutenu dans un premier temps Semir Osmanagić, et le Musée national de Sarajevo, dont les archéologues ont fermement condamné depuis le début ce qu'ils considéraient comme un projet pseudoscientifique. Les dirigeants et spécialistes des deux musées, qui s'étaient déjà opposés par le passé autour du site d'Okolište ${ }^{98}$, et qui se sont heurtés à nouveau lors de la restauration de la forteresse de Visoki ${ }^{99}$, se sont affrontés à propos des "pyramides ", entraînant avec eux les autorités dont ils dépendent, les uns reprochant au musée local son absence de compétence scientifique, les autres reprochant aux autorités fédérales et aux spécialistes de Sarajevo une ingérence dans les affaires du canton et de la municipalité.

51 L'extrême division des pouvoirs et des compétences en Bosnie-Herzégovine, l'absence d'une autorité de régulation dans le domaine de l'archéologie, ont donc permis à Semir Osmanagić de tirer profit des failles du système d'un pays qui n'est toujours pas signataire de la Convention de La Valette de $1992^{100}$ (Convention Européenne pour la Protection du Patrimoine Archéologique), et de bénéficier d'une tolérance, voire d'un encouragement, des autorités, qui seraient difficilement imaginables dans un autre pays d'Europe. Ce phénomène est d'autant plus dommageable, pour les archéologues et défenseurs du patrimoine bosnien, que ce dernier a fait l'objet d'un véritable culturocide pendant le conflit de 1992-1995101, avec la destruction systématique d'éléments patrimoniaux qui accompagnait la politique d'épuration ethnique. Si quelques-uns des éléments les plus emblématiques de ce patrimoine ont pu être sauvés et restaurés, voire, à l'instar du pont de Mostar, reconstruits, on ne compte plus les 
monuments disparus ou menacés de disparition à court terme, et dont le délabrement s'accentue d'année en année, par manque de moyens - et parfois aussi, il faut bien le dire, par manque de volonté politique, sous l'influence de la corruption ou de choix discutables de développement économique ${ }^{102}$. Que le projet pseudo-archéologique de Semir Osmanagić reçoive des financements publics, alors même que le Musée de Sarajevo a vu par deux fois le toit d'une de ses ailes s'effondrer ${ }^{103}$ sur les collections archéologiques par manque d'argent pour des réparations urgentes ${ }^{104}$, ou que les manuscrits survivants de l'incendie de la Bibliothèque de Sarajevo attendent toujours leur restauration dans une caserne désaffectée ${ }^{105}$, a bien de quoi scandaliser.

\section{Conclusion}

L'aventure des "pyramides» de Bosnie, sous ses dehors parfois amusants de projet pseudo-archéologique à mettre au même niveau qu'une affaire de Rennes-le-Château ou une énième découverte de l'Atlantide, est également ainsi un révélateur de certains dysfonctionnements profonds de la société bosnienne : fractures nationales, inefficacité politique, pauvreté... auxquelles il faudrait ajouter les failles du système éducatif, la corruption, l'impuissance ou l'incompétence d'une partie des intellectuels. À la désespérance de la société bosnienne, les " pyramides » peuvent-elles offrir plus qu'une réponse illusoire? Ne sont-elles pas qu'un faux-semblant, dont quelques possibles retombées positives ne compenseront pas les retombées négatives probables: fausse histoire nationale aux implications dangereuses, haine populaire appelée sur les scientifiques d'un pays qui peine déjà à conserver ses diplômés, négation ou détournement d'un patrimoine réel déjà menacé, diffusion massive du charlatanisme sous diverses formes. Que les « pyramides » de Bosnie, après six années de fouilles sans aucun résultat scientifique, continuent d'être visitées et financées par les autorités, et montrées aux enfants des écoles de Bosnie comme un élément de leur patrimoine, ne peut qu'interpeller et préoccuper, au-delà des scientifiques, tous les amoureux de ce magnifique pays.

\section{NOTES}

1. Où se trouverait un mythique trésor templier.

2. Un des sites les plus controversés de l'histoire de l'archéologie, qui a donné lieu à de nombreuses accusations de faux et autres rebondissements, y compris judiciaires, depuis 1924, et qui est un des thèmes favoris des pseudo-archéologues, qui y voient la preuve de l'invention de l'écriture au Paléolithique en Europe. Dans l'état actuel de nos connaissances, il semble que Glozel soit un site authentique, mais gallo-romain et médiéval, ce qui n'exclut pas la possibilité de certains faux contemporains introduits sur le site lors des fouilles des années 20.

3. Stonehenge est bien sûr un site archéologique parfaitement légitime, mais il est un de ceux qui ont donné lieu au plus grand nombre d'interprétations farfelues, tant sur sa fonction que sur les méthodes de sa construction. 
4. Cet impressionnant site pré-colombien de l'Illinois a, comme Stonehenge, fait l'objet de nombreuses interprétations discutables.

5. Cette formation rocheuse sous-marine au large de l'île japonaise de Yonaguni (archipel de Ryukyu) est souvent présentée par les pseudo-archéologues comme un "monument ».

6. Un cas qui aurait pu être quelque peu similaire est celui des "pyramides" de Montevecchia, là aussi des collines présentées par plusieurs auteurs italiens comme des pyramides préhistoriques, voir par exemple Di Gregorio (Vincenzo), Il mistero delle piramidi lombarde [Le mystère des pyramides lombardes], Fermento, 2009; mais aucun de ces auteurs n'a encore réussi à faire accepter ses idées par les autorités italiennes, ni à faire financer une campagne de fouilles.

7. Emrić (Amel), «Experts Find Evidence of Bosnia Pyramid », The Associated Press, jeudi 20 avril 2006 - $\quad$ http://www.washingtonpost.com/wp-dyn/content/article/2006/04/20/ AR2006042001800.html

8. Woodard (Colin), "The Mystery of Bosnia's Ancient Pyramids", Smithsonian Magazine, décembre 2009 - http://www.smithsonianmag.com/history-archaeology/The-Mystery-ofBosnias-Ancient-Pyramids.html?c=y\&page $=1$

9. Nukić (Nadija), Report on a geological survey of Visočica elevation in Visoko, Bosnia and Herzegovina, Sarajevo, novembre 2005 - http://www.bosnianpyramid.com/PDFS/egeoloskiVisočicaokt2005.pdf 10. Osmanagić (Semir), Bosanska piramida Sunca : Otkriće prve evropske piramide [La pyramide bosnienne du Soleil : découverte de la première pyramide européenne], Sarajevo : Klepsidra, 2005 - http://www.alternativnahistorija.com/AH8main.htm

11. Avant même le début des fouilles, un âge très avancé pour les "pyramides » est affirmé par Semir Osmanagić : plusieurs milliers d'années, voire, dans son ouvrage Bosanska Piramida Sunca de 2005, 12000 ans.

12. Exclusive interview with Semir Osmanagić, BosnianPyramids.org - http:// www.bosnianpyramids.org/index.php?id=6\&lang=en

13. Markey (Sean), «Pyramid in Bosnia - Huge Hoax or Colossal Find?", National Geographic News, 12 mai 2006 - http://news.nationalgeographic.com/news/2006/05/pyramid-bosnia-1.html 14. Voir le site de la Fondation créée par Semir Osmanagić : http://piramidasunca.ba/

15. Rose (Mark), «The Bosnia-Atlantis Connection », Archaeology Online, 27 avril 2006 - http:// www.archaeology.org/online/features/Osmanagić/index.html

16. Harding (Anthony), "That Bosnian pyramid!", The European Archaeologist, (25), été 2006 http://www.e-a-a.org/TEA25.pdf

17. Site personnel de Semir Osmanagić, où ses livres sont disponibles en ligne: http:// www.alternativnahistorija.com/

18. Courrier de Grace Fegan, http://allaboutbosnianpyram.blogger.ba/arhiva/ 2006/05/09\#241665

19. Bačanović (Vuk), «U potrazi za izgubljenim izvještajima [A la recherche des rapports perdus]», BH Dani, (552), 11 janvier 2008 - http://www.bhdani.com/default.asp? kat=txt\&broj_id=552\&tekst_rb=12. Traduction disponible ici: http://irna.lautre.net/A-larecherche-des-rapports-perdus.html

20. Harding (Anthony), « The great Bosnian pyramid scheme », British Archaeology, (92), janvierfévrier 2007 - http://www.britarch.ac.uk/ba/ba92/feat3.shtml

21. Cité dans Rose (Mark), art.cit..

22. "Bosnian pyramids»: A pseudoarchaeological myth and a threat to the existing cultural and historical heritage of Bosnia-Herzegovina, lettre au Directeur général de l'UNESCO Koichiro Matsuura, 12 juin 2006 - http://www.archaeology.org/online/features/Osmanagić/UNESCO.pdf

23. Vrabac (Sejfudin),et al., Izvještaj o geološkim istraživanjima Visočice kod Visokog [Rapport sur la géologie de Visočica près de Visoko], Rudarsko-geološko-građevinski Fakultet Univerziteta u Tuzli, 17 avril 2006 - http://irna.lautre.net/IMG/pdf/Output-2.pdf

24. Bačanović (Vuk), art.cit. 
25. Paul Heinrich's geological postings on the "Bosnian Pyramid » sur le forum de discussion " Hall of Maat » - http://www.hallofmaat.com/modules.php?name=Articles\&file=article\&sid=103

26. Schoch (Robert M.), «The Bosnian Pyramid Phenomenon », The New Archaeology Review, 1 (8), septembre $2006 \quad$ - http://www.robertschoch.net/ Bosnian\%20Geology\%20Robert\%20Schoch\%20\%20Pyramids\%20Colette\%20Dowell.htm

27. Osmanagić (Semir), op.cit.

28. «MRAV » : Međunarodna Radna Akcija Volontera [Chantier international de volontaires]

29. Par exemple le forum de David Icke : http://forum.davidicke.com/index.php

30. La Fondation a d'ailleurs fréquemment tendance à enjoliver le $\mathrm{CV}$ des participants aux fouilles; par exemple, un jeune Italien titulaire d'une licence en archéologie est régulièrement présenté comme « le Docteur X, archéologue »; il est vrai que le titre de « Dottore » est beaucoup plus largement attribué en Italie que dans les autres pays d'Europe.

31. http://www.bosnic.com/

32. Un exemple: «Mekki Torabi posjetio bosanske piramide [Mekki Torabi a visité les pyramides de Bosnie]», Metro Portal, 9 novembre 2010 - http://metro-portal.hr/mekki-torabi-posjetiobosanske-piramide/50966. Mekki Torabi est un guérisseur marocain.

33. "Semir Osmanagić a Damanhur [Semir Osmanagić à Damanhur] ", La Voce del Canavese, 16 avril 2007 - http://www.damanhur.info/attachments/1628_mag000450001.pdf

34. Voir «De la transparence et de l'intégrité scientifique », Le site d'Irna, 11 octobre 2008 http://irna.lautre.net/De-la-transparence-et-de-l.html

35. Bačanović (Vuk), «Osmanagić je otjerao stručnjake iz projekta » [Osmanagić a chassé les scientifiques du projet], BH Dani, (546), 30 novembre 2007 - http://www.bhdani.com/default.asp? kat=txt\&broj_id=546\&tekst_rb=25

36. Voir par exemple cette explication fournie par un volontaire sur le forum de David Icke: http://forum.davidicke.com/showpost.php?p=1060063674\&postcount=65

37. Page consacrée à Okolište sur le site du Deutsche Archäologische Institut: http:// www.dainst.org/de/node/24076? ft=all

38. Anđelić (Pavao), Visoko i okolina kroz historiju [Visoko et ses environs à travers l'histoire], Visoko, 1984

39. Old Visoki fort, the historic site, sur le site de la Commission pour la Protection des Monuments Nationaux : http://www.kons.gov.ba/main.php?id_struct=50\&lang=4\&action=view\&id=2409

40. Osmanagić (Semir), Otvoreno pismo prof. Enveru Imamoviću [Lettre ouverte au professeur Enver Imamović], 8 mai 2006 - http://www.piramidasunca.ba/ba/index.php/20060508233/index.php? option=com_content\&view=article\&id=233:semir-Osmanagić-otvoreno-pismo-prof-enveruimamovicu-koji-u-niz-navrata-uporno-iznosi-neistine-o-p\&catid=1:najnovije\&Itemid=126 41. Voir « La forteresse qui n'existait pas », Le site d'Irna, 21 juillet 2008 : http://irna.lautre.net/ La-forteresse-qui-n-existait-pas.html

42. Voir en particulier Bešlagić (Šefik), Stećci, kultura i umjetnost [Stećci, culture et art], Sarajevo, 1979.

43. Bačanović (Vuk), « Osmanagić je otjerao stručnjake iz projekta » (art.cit.).

44. Djurdjevic (Nenad), In the name of the Sun, and the Moon, and the Stars, 25 octobre 2009 http://www.bosnian-pyramid.com/journal/2009/10/25/in-the-name-of-the-sun-and-the-moonand-the-stars.html

45. Vrbančić (Davorin), Bosanske piramide na stećcima [Les pyramides de Bosnie sur les stećci], 27

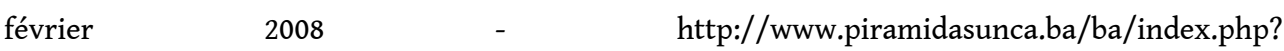
option=com_content\&view=article\&id=1640:bosanske-piramide-na-

steima\&catid=1:najnovije\&Itemid=126

46. Bohannon (John), «Mad About Pyramids», Science, 313, 22 septembre 2006 - http:// www.johnbohannon.org/NewFiles/bosnia.pdf 
47. Djurdjevic (Nenad), Archaeologists Unearth Votive Pyramid in Visoko! , 29 septembre 2008 http://bpblognews.blogspot.com/2008/09/archaeologists-unearth-votive-pyramid.html

48. Voir le communiqué de presse de l'Université de Kiel où l'artefact est actuellement en cours d'étude : http://www.uni-kiel.de/aktuell/pm/2008/2008-093-tonobjekt.shtml

49. Voir «Le bout du tunnel ? ", Le site d'Irna, 16 août 2011 - http://irna.lautre.net/Le-bout-dutunnel.html

50. Osmanagić (Semir) et al., Projekt istraživanja i konzervacije Starog grada Visoki [Projet de recherche et conservation de la Vieille Ville de Visoko], Sarajevo, avril 2007 - http:// piramidasunca.ba/en/index.php?option=com_content\&task=view\&id=747\&Itemid=26

51. L'expression a été utilisée pour la première fois par le géologue américain Paul Heinrich dans ce post : http://www.hallofmaat.com/read.php?1,405378,405510,sv=1\#msg-405510

52. Schoch (Robert M.), "Politics, Money and Science", Atlantis Rising, (85), janvier 2011 http://www.innoventek.com/PoliticsMoneyAndScience_AtlantisRisingNbr85Jan2011.pdf

53. Osmanagić (Semir) et al., Izvještaj o Programu istraživanja Fondacije AP BPS za 2006. godinu [Rapport sur le programme de fouilles de la Fondation AP BPS pour l'année 2006], Sarajevo, février $\quad 2007 \quad$ - $\quad$ http://www.piramidasunca.ba/ba/index.php? option=com_content\&view=article\&id=651:izvjetaj-o-programu-istraivanja-za-2006-

godinu\&catid=23:izvjetaji\&Itemid=61

54. Osmanagić (Semir), Netehnološka Civilizacija Maja naspram Modernih tehnoloških Civilizacija [La civilisation non technologique des Mayas par rapport aux civilisations technologiques modernes], thèse de sociologie soutenue auprès de la Faculté des Sciences Politiques de l'Université de Sarajevo, mai 2009 - http://www.semirOsmanagić.com/ba/Maje__doktorska_disertacija_jun_2009.doc

55. http://piramidasunca.ba/bs/aktuelnosti/icbp/icbp-2011/itemlist/category/14icbp-2008.html

56. http://piramidasunca.ba/bs/aktuelnosti/icbp/icbp-2011

57. Program of the First International Scientific Conference Bosnian Valley of the Pyramids, Sarajevo, 25 au $\quad 30 \quad$ août $2008 \quad-\quad$ http://www.bosnianpyramid.com/PDFS/ICBP\%20\%20Program\%20July\%2016th\%202008.pdf

58. La « hiérolinguistique » est la « science des langues sacrées». Toutefois, la seule mention de cette science sur Internet apparaît sur des pages de M. Stekel, ou consacrées à cet auteur...

59. Barakat (Aly A.), Geological and Geo-archaeological Observations on the Bosnian Pyramids in Visoko, Le Caire, juin 2006 - http://www.piramidasunca.ba/en/en/news/1004-the-geological-and-geoarchaeological-observations-on-the-bosnian-pyramids-in-visoko.html

60. Swelim (Nabil), The Pyramid Hills : Visočica and Plješevica Hrašće - Observations, and Analyses, septembre 2007 - http://www.nabilswelim.com/downloads/Pyr_Hills.pdf.pdf

61. Swelim (Nabil), Visočica on the balance, 2010 - http://www.nabilswelim.com/downloads/ vic_10.pdf

62. Swelim (Nabil), Originalno pismo dr. Nabila Swelima dr. Blagoju Govedarici [Lettre originale du Dr. Nabil Swelim au Dr. Blagoje Govedarica], 7 mai 2009 - http://www.piramidasunca.ba/ba/index.php? option=com_content\&view=article\&id=2321:swelim-govedarici-vi-ste-arogantni-i-nemate-nauihkvalifikacija-da-govorite-o-piramidama\&catid=1:najnovije\&Itemid=126

63. Nikolić (Domagoj), Razgovori s piramidom [Discussions avec une pyramide], 6 septembre 2011 http://piramidasunca.ba/bs/iz-mog-ugla/item/83-razgovori-s-piramidom.html

64. http://www.salviamoci2012.it/

65. http://www.sbresearchgroup.eu/

66. Robins (Don), Circles of Silence, Souvenir Press, 1985.

67. Fil de discussion Piramidi in Europa sur le forum archéologique Ostraka, http:// ostraka.forumfree.it/?t=53186252 
68. Pruitt (Tera C.), Authority and Archaeology: An Analysis of the Role of Authority in the Production of Archaeological Accounts of the Past (titre provisoire), thèse de doctorat de l'Université de Cambridge, à paraître.

69. Italijanski Institut potvrðuje postojanje vještačkog betona na Bosanskoj Piramidi Sunca [Un Institut italien confirme l'existence de béton artificiel sur la Pyramide bosnienne du Soleil], 21 décembre 2009 http://www.piramidasunca.ba/ba/index.php?

option=com_content\&view=article\&id=2594:italijanski-institut-potvrje-postojanje-vjetaogbetona-na-bosanskoj-piramidi-sunca\&catid=1:najnovije \&Itemid $=126$

70. Građevinski institut Tuzla, Preliminarni rezultati ispitivanja uzoraka materijala uzetih sa Piramide Sunca i Piramide mjeseca [Résultats préliminaires de l'examen d'échantillons de matériaux prélevés sur la Pyramide du Soleil et la Pyramide de la Lune], 12 janvier 2007 - http:// piramidasunca.ba/bs/projekat/Izvještaji/179-preliminarni-rezultati-ispitivanja-uzorakamaterijala-uzetih-sa-piramide-sunca-i-piramide-mjeseca-2.html

71. Pašić (Muhamed), Rezultati ispitivanja uzoraka Bosanske piramide Sunca [Résultats de l'examen d'échantillons de la Pyramide bosnienne du Soleil], 26 janvier 2009 - http:// www.piramidasunca.ba/ba/index.php/200901262159/index.php?

option=com_content\&view=article\&id=2159:analiza-betonskog-materijala-s-

piramida\&catid=1:najnovije\&Itemid $=126$

72. "Tunnels, bois fossile et carbone 14 », Le site d'Irna, 21 septembre 2008 - http:// irna.lautre.net/Tunnels-bois-fossile-et-carbone-14.html

73. Le "culte du cargo", ou « cargo cult", renvoie à des comportements développés dans les sociétés pré-industrielles du Pacifique durant et après la deuxième guerre mondiale, reposant sur des pratiques de mimétisme ; le physicien Richard Feynman a repris l'expression de "Cargo Cult Science » pour désigner toutes les variantes de science approximative et de pseudoscience.

74. Coppens (Philip), « The New Pyramid Age - Unearthing Europe's Oldest Pyramid Complex », Nexus Magazine, 17 (1), décembre 2008-janvier 2009 - http://www.philipcoppens.com/ nap_art4.html

75. Osmanagić (Semir), «How did "Pharaoh" Dr. Zahi Hawass try to stop Bosnian Pyramids project», The New Era Times, mars 2011 - http://www.tnetimes.com/article/244-verge-falldictator-among-egyptologists-zahi-hawass-edgar-cayce-pyramid-sphinx-bosnia-semir-dr-samOsmanagić-Osmanagićh-corruption-egypt-mubarak-antiquities-regime-suzanne-nationalgeographic-history-channel-bouval-hancock/

76. Osmanagić (Semir), od masona do mentalnih projekcija [Des francs maçons aux projections mentales], non publié - Disponible en ligne sur le site de l'auteur: http:// www.alternativnahistorija.com/AH1.htm

77. Bohannon (John), art.cit.

78. Par exemple le fait que la longueur des arêtes de Visočica serait de 365 mètres exactement, fait auquel on donne une signification astronomique : Lazović (T.), «Sjeverna strana piramide Sunca je jednakostranični trougao [La face Nord de la pyramide du Soleil est un triangle équilatéral]», Dnevni Avaz, 17 avril 2006

79. Exemple : le « Polycontrast Interference Photography » de Harry Oldfield, voir « Vérifications pseudo-scientifiques », Le site d'Irna, 2 mai 2007 - http://irna.lautre.net/Verifications-pseudoscientifiques.html

80. Ce parc d'attraction, consacré aux mystères archéologiques et aux extraterrestres, et fondé par l'écrivain von Däniken en Suisse, a fermé ses portes en novembre 2006.

81. Ainsi, une conférence de presse annonçant une validation des théories de Semir Osmanagić par le Dr Swelim et ses collègues égyptiens dès le lendemain de leur arrivée à Sarajevo en septembre 2007 ne donne lieu qu'à une seule question des journalistes présents, portant sur la durée du séjour des scientifiques égyptiens.

82. Harding (Anthony), art.cit. 
83. Foer (Joshua), «Love Triangles ", Outside online, 24 avril 2007 - http://outsideonline.com/ outside/destinations/200705/bosnia-herzegovina-1.html

84. Marcus (Alexander), Fotini (Christia), «Walk Like an Egyptian : Identity Construction in Post-Conflict Bosnia-Herzegovina », Comparative Politics (à paraître).

85. Et peut-être aussi comme l'équivalent bosniaque de Medjugorje, le grand pèlerinage catholique d'Herzégovine, qui attire bon an mal an deux millions de pèlerins dans cette petite ville?

86. Tricic (Lejla), Dispatches from the Bosnian Valley of the Kings or How Bosnians created a fictionalized national History, thèse de Master, California State University, Fresno, décembre 2007 http://ecollections.lib.csufresno.edu/specialcollections/document.php?CISOROOT=/

thes\&CISOPTR $=19840 \&$ CISOSHOW $=19713$

87. Voir par exemple ce message de forum: http://ostraka.forumfree.it/? $\mathrm{t}=53186252 \& \mathrm{st}=30$ \#entry 453062181

88. Osmanagić (Semir), Bosanska piramida Sunca... (op.cit.), voir le chapitre I, «Anthropologie génétique ».

89. Holtorf (Cornelius), « Meta-stories of archaeology », World Archaeology, 42 (3), 2010 - http:// www.tandfonline.com/doi/abs/10.1080/00438243.2010.497382

90. Voir en particulier Kolstø (Pål), « Procjena uloge historijskih mitova u modernim društvima [Evaluation du rôle des mythes historiques dans les sociétés modernes]», in Historijski mitovi na Balkanu, Sarajevo: Institut za Istoriju, 2003 - http://www.iis.unsa.ba/izdavacka_djelatnost/ posebna_izdanja/mitovi/mitovi.html

91. Harding (Anthony) et al., "Fictitious pasts : a danger for European archaeology? ", 14 ème conférence annuelle de l'Association des Archéologues Européens, Malte, 2008 - http:// events.um.edu.mt/eaa2008/harding.pdf

92. Voir http://irna.lautre.net/Ce-que-les-institutions,107.html, courrier de la Commission pour la Protection des Monuments Nationaux au ministre Grahovac, 17 avril 2007

93. Voir http://irna.lautre.net/Ce-que-les-institutions.html, courrier du Zemaljski Muzej au ministre Grahovac, 25 avril 2007

94. http://irna.lautre.net/Ce-que-les-institutions.html, courrier du Conseil Fédéral pour la Géologie au ministre Grahovac, 20 avril 2007

95. http://irna.lautre.net/Ce-que-les-institutions.html, courrier de l'Académie des Sciences et Arts de Bosnie-Herzégovine au ministre Grahovac, 23 avril 2007

96. http://irna.lautre.net/Ce-que-les-institutions,107.html, courrier du ministre Gavrilo Grahovac au gouvernement de la Fédération, 9 mai 2007

97. Décisions de la Commission pour la Protection des Monuments Nationaux n05.2-2-1047/03-12 du 15 mars 2006, et n05.1-02-106/09-2 du 10 mars 2009 - http:// www.kons.gov.ba/main.php?id_struct=50\&lang=1\&action=view\&id=2409

98. Center for Investigative Reporting, BiH archeological treasure neglected through lack of will and leadership, publication en ligne du CIN (Centar za istraživačko novinarstvo), Sarajevo, 2004 http://www.cin.ba/Stories/P2_Culture/?cid=400,1,1

99. «Ignominies », Le site d'Irna, 12 août 2008 - http://irna.lautre.net/Ignominies.html

100. Lettre ouverte de la communauté scientifique bosnienne à M. Christian Schwarz-Schilling, Haut Représentant de l'ONU en Bosnie-Herzégovine, Sarajevo, 14 mars 2007 - http://irna.lautre.net/IMG/ pdf/lettre_schwarz01.pdf et version anglaise: http://irna.lautre.net/IMG/pdf/letter-ohrfinal02.pdf

101. Wallon (Emmanuel), « Portrait de l'artiste en témoin - Les guerres yougoslaves de la page à l'écran ", in Lescot (David), Véray (Laurent), éds., Les mises en scène de la guerre au XXe siècle, Théâtre et cinéma, Paris: Nouveau Monde, 2011 - http://e.wallon.free.fr/IMG/pdf/Artistetemoin.pdf 
102. Conseil de l'Europe, Programme régional pour le patrimoine culturel et naturel du Sud-Est de l'Europe: Bosnie-Herzégovine, 2010 - http://www.coe.int/t/dg4/cultureheritage/cooperation/see/ countries/bosnia_FR.asp?

103. Bačanović (Vuk), «Državne institucije podržavaju Osmanagićevu sektu » [Les institutions d'Etat soutiennent la secte d'Osmanagić], interview publiée dans BH Dani, (496), 15 décembre 2006 - http://www.bhdani.com/default.asp?kat=txt\&broj_id=496\&tekst_rb=19

104. Sadiković (Mirna), «Sedam bh. institucija kulture pred zatvaranjem » [Sept institutions culturelles bosniennes menacées de fermeture], Tuzlanski Info Portal, 1 juillet 2011 - http:// www.tip.ba/2011/07/01/sedam-bh-institucija-kulture-pred-zatvaranjem/

105. Arte, Vidéo: La Bibliothèque de Sarajevo, mai 2011 - http://www.arte.tv/fr/ $2151166, \mathrm{CmC}=2946150 . \mathrm{html}$

\section{RÉSUMÉS}

Cet article propose une synthèse critique sur les pyramides qu'aurait découvertes Semir Osmanagić en Bosnie-Herzégovine. Cette affaire de pseudo-archéologie s'explique selon un contexte géomorphologique, culturel et social complexe qui s'articule autour de la personnalité de son inventeur.

This article provides a critical synthesis around the pyramids discovered by Semir Osmanagić in Bosnia. This pseudo-archeology affair can be explained by its complex context in three different extents: geomorphologic, cultural and social, joined with Osmanagić's personality.

INDEX

Keywords : Pyramids, Bosnia-Herzegovina, Semir Osmanagić, pseudo-archeology

Mots-clés : pyramides, Bosnie-Herzégovine, Semir Osmanagić, pseudo-archéologie

\section{AUTEUR}

\section{IRNA}

fr 